\title{
Adaptation of Saccades and Perceived Size after Trans-Saccadic Changes of Object Size
}

\author{
(Di)Annalisa Bosco, ${ }^{1}$ Markus Lappe, ${ }^{2}$ and $\odot$ Patrizia Fattori ${ }^{1}$ \\ ${ }^{1}$ Department of Pharmacy and Biotechnology, University of Bologna, 40126 Bologna, Italy, and ${ }^{2}$ Department of Psychology, University of Munster, D-48149 \\ Munster, Germany
}

When saccadic eye movements consistently fail to land on the intended target, saccade accuracy is maintained by gradually adapting the amplitude of successive saccades to the same target. Such saccadic adaptation is usually induced by systematically displacing a small visual target during the execution of the saccade. However, saccades are normally performed to extended objects. Here we report changes in saccade amplitude when the size of a target object is systematically changed during a saccade. Moreover, we find that this manipulation also affected the visual perception of the size of that object. Human subjects were tested in shortening and lengthening adaptation where they had to make saccades to targets of different sizes, which were each shortened or lengthened during saccade execution, respectively. In both experiments, a preadaptation and postadaptation phase required manually indicating the horizontal size of each target by grip aperture and, in a further experiment, a verbal size report. We evaluated the effect of change in visual perception on saccade and on the two modalities of judgment. We observed that (1) saccadic adaptation can be induced by modifying target object size and (2) this gradual change in saccade amplitude in the direction of the object size change evokes a concomitant change in perceived object size. These findings suggest that size is a relevant signal for saccadic system and its trans-saccadic manipulation entails considerable changes at multiple levels of sensorimotor performance.

Key words: eye movements; oculomotor control; perception; vision

\section{Significance Statement}

Across a saccade to an object the visual system receives two views of that object, a presaccadic peripheral view and a postsaccadic foveal view. We show that manipulation of the size of the object after the saccade modifies presaccadic size perception and motor control. Saccade amplitudes become smaller when the postsaccadic object is smaller and perceived size estimates become smaller, too. The reverse is true when the object is larger after the saccade. These findings suggest that an object feature as size is a relevant signal that influences the saccadic system and the size distortion following saccadic adaptation indicates that the representation of the central part of visual field is critical for interaction between hand and object or object recognition.

\section{Introduction}

Our brain explores the external world by saccadic eye movements. Saccades involve the visual system to localize a target and the motor system to activate the eye muscles. The visuomotor process of saccade control needs to be plastic. This plasticity is

\footnotetext{
Received Jan. 12, 2015; revised Sept. 1, 2015; accepted Sept. 3, 2015.

Author contributions: A.B., M.L., and P.F. designed research; A.B. performed research; A.B. analyzed data; A.B., M.L., and P.F. wrote the paper.

This work was supported by Ministero dell'Università e della Ricerca, Futuro in Ricerca 2013, Fondazione del Monte di Bologna e Ravenna, Deutsche Forschungsgemeinschaft LA952-6, and University of Bologna's Institute of Advanced Studies. We thank Giacomo Placenti for support in setting up the experimental apparatus and Kerstin Richert for helping in data collection.

The authors declare no competing financial interests.

Correspondence should be addressed to Dr. Patrizia Fattori, Department of Pharmacy and Biotechnology, University of Bologna, Piazza di Porta San Donato 2, 40126 Bologna, Italy. E-mail: patrizia.fattori@unibo.it.

DOI:10.1523/JNEUROSCI.0129-15.2015

Copyright $\odot 2015$ the authors $\quad 0270-6474 / 15 / 3514448-09 \$ 15.00 / 0$
}

commonly studied by means of saccadic adaptation, a learning mechanism that ensures saccade accuracy in the face of changes in motor dynamics due to daily fatigue, aging, or pathology. Typically, research on oculomotor control uses small targets (a point or cross), and sensory-motor transformation depends on establishing a simple correspondence between visual and motor coordinate systems. Thus, a visual stimulation at a given retinal position gives rise to a motor command of appropriate direction and amplitude. However, the vast majority of objects in natural environments are spatially extended. When such a stimulus is selected by the saccadic system, the eyes generally land near its center, suggesting that the representation of a central reference position within the spatially extended stimulus is used to guide saccades (Kowler and Blaser, 1995). Despite much research of saccadic adaptation for targeting-saccades (for review, see Hopp and Fuchs, 2004; Pélisson et al., 2010), little is known about such a mechanism for saccades toward targets of different spatial ex- 
A

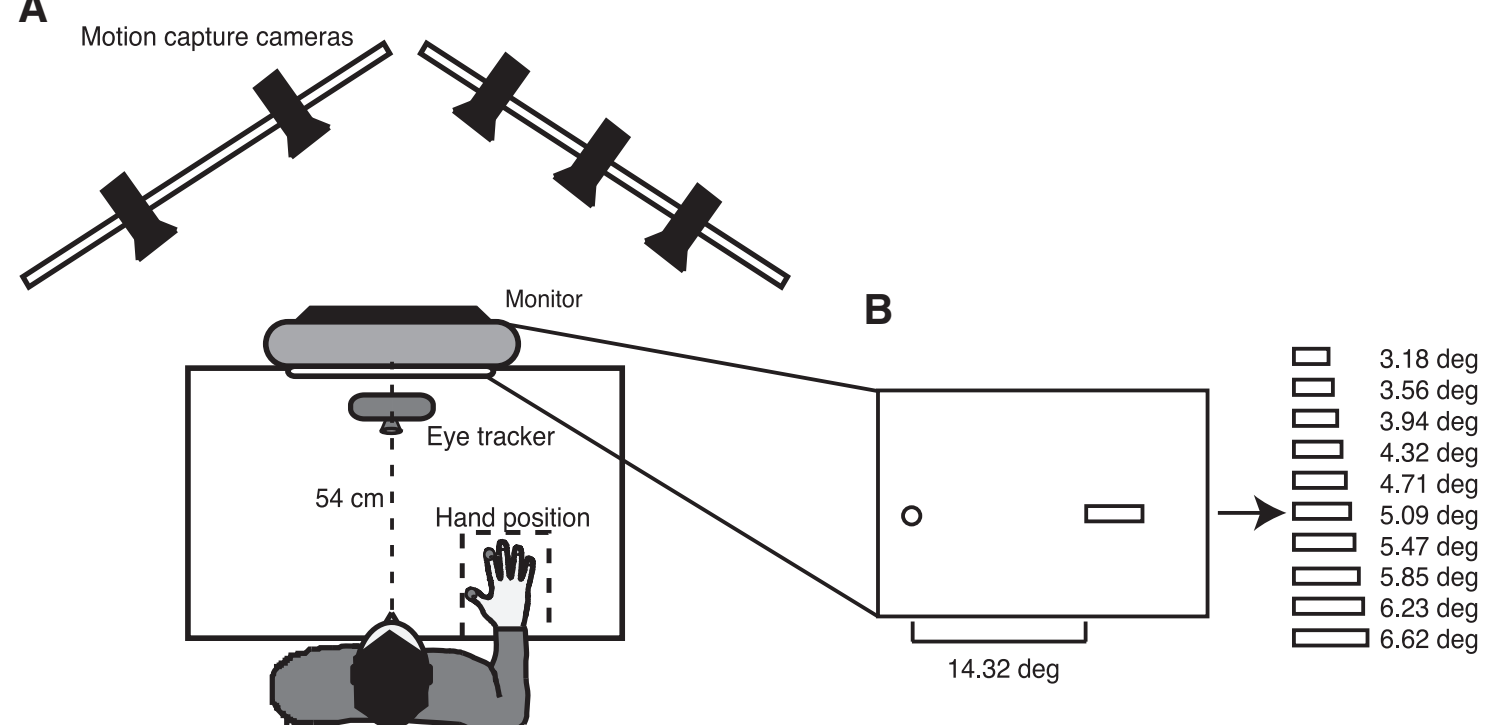

C

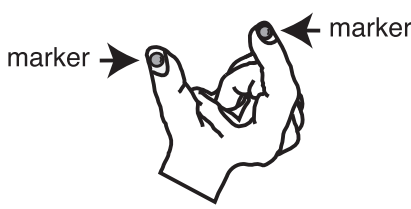

Figure 1. Experimental setup and stimuli. $\boldsymbol{A}$, Top view of the experimental setup. An infrared-based eye-tracker was used to measure the horizontal position of the dominant eye. An optical motion capture system recorded the 3D positions of the thumb and index tips. Stimuli were projected on a computer monitor at $54 \mathrm{~cm}$ from the subject's eyes and hand position rested at $34 \mathrm{~cm}$ from the body midline. $\boldsymbol{B}$, Frontal view of the stimuli used in the experiments: circle represents fixation target ( 0.3 degree of radius); rectangles represent saccade and size estimation targets. $\boldsymbol{C}$, Typical hand posture during the size estimation phase and passive markers (10 $\mathrm{mm}$ of radius) attached on the thumb and index nails.

tent. In studies on reading, researchers have identified withinobject saccades and established the preponderant role of word size on the probability of making more than one fixation on words (McConkie et al., 1989). In that context, saccade amplitude increases with word length, suggesting that object size is a crucial parameter to execute within-object saccades. A study performed on within-object saccades explored the possibility to adapt eye movement to a systematic change in object size (Lavergne et al., 2010). Increasing or decreasing object size during within-object saccade execution showed results where within-object saccade amplitude was lengthened and shortened, respectively. However, the encoding of object size in saccadic adaptation of the primary saccade to spatially extended stimuli is not clear.

Recent literature has explored the possibility that the saccadic system shares common coordinates with other domains. Several researches have demonstrated that the modification of motor variables by saccade adaptation leads to a distortion of visual localization of the target executed by hand pointing or by perceptual reports (Bahcall and Kowler, 1999; Awater et al., 2005; Bruno and Morrone, 2007; Collins et al., 2007, Zimmermann and Lappe, 2010; Garaas and Pomplun, 2011; Gremmler et al., 2014). All these studies imply that localization of visual stimuli is based on a perceptual representation that is linked to an action representation that guides saccades and pointing. If a similar link exists for object size perception, then a trans-saccadic alteration to object size could affect both saccade targeting and visual size perception. In the present study, using an original approach to induce saccadic adaptation with extended objects, we addressed two questions: how the saccadic system processes the size signal of targets to correct eye movements in both shortening and lengthening paradigms and whether object size perception is also changed.

\section{Materials and Methods}

Participants. A total of 35 right-handed subjects (24 females and 11 males, ages 19-40 years) with normal or corrected-to-normal vision took part in the experiments. Fourteen participants completed Experiment 1 (manual shortening experiment), 10 participants completed Experiment 2 (manual lengthening experiment), and 11 participants were involved in a verbal report control experiment with both lengthening and shortening conditions. The subjects had no history of musculoskeletal or neurological disorders. All subjects were naive to the experimental purpose of the study and gave informed consent to participate in the experiment. Procedures were approved by the Bioethical Committee of the University of Bologna and the Ethics Commission of the Department of Psychology of the University of Muenster and were in accordance with the Declaration of Helsinki.

Apparatus and setup. Participants in Experiments 1 and 2 were seated in an environment with dim background lighting and viewed a touchscreen monitor (ELO IntelliTouch, 1939L), which displayed target stimuli within a visible display of $37.5 \times 30.0 \mathrm{~cm}$. To stabilize head position, the participants placed their heads on a chin rest located $54 \mathrm{~cm}$ from the screen, which resulted in a visual field of $39.8 \times 31.8 \mathrm{deg}$ (Fig. $1 A$ ). The display had a resolution of $1152 \times 864$ pixels and a frame rate of $60 \mathrm{~Hz}$. For stimulus presentation, we used MATLAB (The MathWorks) with the Psychophysics toolbox extension (Brainard, 1997). The stimuli were blue, green, and red dots with a radius of 0.3 deg and 10 differently sized red horizontal bars (size in deg: 3.18, 3.56, 3.94, 4.32, 4.71, 5.09, 5.47, $5.85,6.23,6.62)$ as it is reported in Figure $1 B$. Eye movements were monitored by the EyeLink 1000 system (SR Research), which sampled gaze positions with a frequency of $1000 \mathrm{~Hz}$. Viewing was binocular, but the dominant eye was recorded. The calibration was performed with 


\section{A Manual estimation phase}

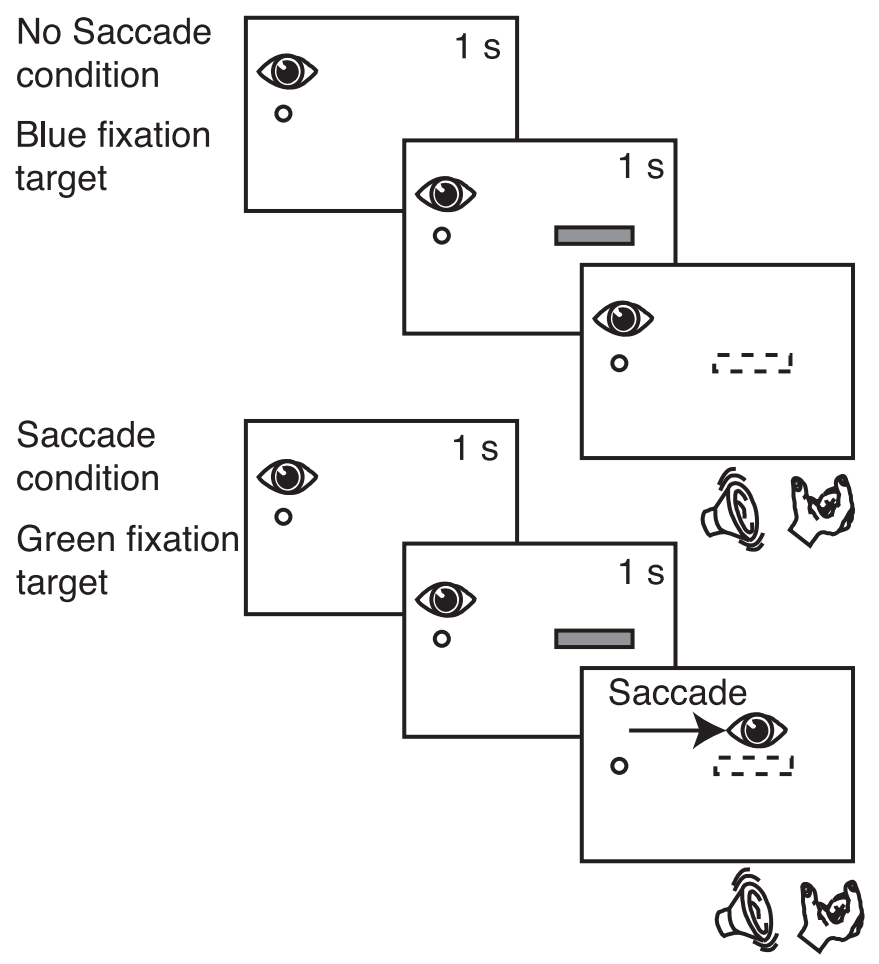

\section{B Saccade adaptation phase}



Figure 2. Task design. $\boldsymbol{A}$, Manual estimation phase. Top row, No-saccade condition trial. Subjects were instructed to fixate at the blue fixation target shown as a small circle. After $1 \mathrm{~s}$, a bar was flashed (gray rectangle) for $1 \mathrm{~s}$; and following an acoustic signal, subjects had to indicate (while keeping fixation) the perceived size of the bar by the grip aperture. Bottom row, Saccade condition trial. Subjects fixated at the green fixation target. After $1 \mathrm{~s}$, a bar was projected; and when an acoustic signal was activated, subjects were required to perform a saccade toward the bar and indicate the perceived size of the bar. As soon as the saccade was detected, the bar disappeared from the screen. $\boldsymbol{B}$, Saccade adaptation. Top row, Shortening adaptation phase. At the beginning of the trial, the red fixation target was presented and the subjects' gaze was directed toward it. After $1 \mathrm{~s}$, a red bar appeared, but subjects had to continue to fixate the fixation target. After a randomized time, an acoustic signal informed them to execute a saccade toward the bar. As soon as the saccade was detected, the bar was decreased in size by $30 \%$ of its length. Bottom row, Lengthening adaptation phase. It was structured in the same way of shortening adaptation phase with the difference that the bar was increased in size by $30 \%$ during saccade execution.

EyeLink software at the beginning of each recording session using a 9 point calibration grid that allowed precise measurements of horizontal and vertical eye position. Hand position was measured by a motion capture system (VICON, 460; frequency of acquisition $100 \mathrm{~Hz}$ ), which follows the trajectory of the hand in three dimensions by recording infrared light reflection on passive markers placed on the index and thumb fingers, respectively (Fig. 1C). The hand was kept on the table at $34 \mathrm{~cm}$ from the body midline within a square on the table marked with tape and detectable by touch (Fig. 1A). All markers were held in place on the participant skin with small pieces of adhesive tape that allowed freedom of movement of the hand and fingers.

Behavioral task. In Experiment 1, participants were tested in three successive phases: preadaptation manual estimation phase, shortening adaptation, and postadaptation manual estimation phase. In the preadaptation and postadaptation manual estimation phases, participants performed 20 trials in which they were required to manually indicate the horizontal size of a red bar presented 14.32 deg on the right of the initial fixation target. The sequence of events of the preadaptation manual estimation phase is illustrated in Figure $2 A$. The phase consisted of two randomized conditions distributed in 20 trials where the fixation target could be blue or green and called no-saccade and saccade condition, respectively. If the blue fixation target appeared on the screen, subjects were required to fixate it. After $1 \mathrm{~s}$, a red bar was presented for $1 \mathrm{~s}$. Thereafter, an acoustic signal informed them to manually indicate the perceived size of the bar by extending the thumb and index finger, but keeping the fixation on the blue fixation target. If the fixation target was green, subjects looked at it and after $1 \mathrm{~s}$ a red bar was presented on the screen. Subjects remained with the gaze on the green fixation target for $1 \mathrm{~s}$ more, and then an acoustic signal notified them to perform a saccade toward the bar and to manually indicate the perceived size of the bar. The bar was extinguished as soon as saccade onset was detected. All participants indicated the size of the bars at the same hand position inside the hand position square to avoid any effect of distance on the estimation of size and distance between subject eyes and the screen was kept constant to obtain the same vergence angle.

In the saccadic adaptation phase of Experiment 1 (Fig. $1 A$ ), we induced a shortening adaptation using a double-step protocol with delayed saccades where we manipulated the size of the target bars while the saccade was in flight. The trial sequence is shown in Figure $2 B$ (top). For the first 20 trials (preadaptation trials), one of ten horizontal red bars (as shown in Fig. $1 B$ ) was presented at 14.32 deg on the right of fixation target. Bar presentation occurred $1 \mathrm{~s}$ after fixation target appearance. Participants were required to keep fixation on the initial target for 1-1.8 s until an acoustic signal informed them to perform a saccade to the bar. For the remaining 180 trials (adaptation trials), the sequence of events was the same but the bar was shortened by $30 \%$ of its original length during saccade execution (Fig. 2B, top). Saccade onset was detected when the eye movement exceeded a distance of 2 deg from the fixation target. As soon as the saccade onset was detected, the bar was decreased to a new size to induce a postsaccadic error. After the amplitude was successfully altered in the adaptation trials, we again tested the participants in the postadaptation manual estimation phase (Fig. 2A). This phase was structured identically to the preadaptation manual estimation phase with randomization of no-saccade and saccade conditions. During estimation phases, participants had no visual feedback of the hand because they maintained their gaze on the stimuli projected on the screen.

Experiment 2 was identical to Experiment 1, except for the adaptation session. During this session, the adaptation trials consisted in lengthening the bar by $30 \%$ of its length. Because lengthening adaptation is slower than shortening adaptation (Ethier et al., 2008; Golla et al., 2008; 
Panouillères et al., 2009), we decided to use different numbers of adaptation trials in the two experiments. Thus, to induce significant lengthening adaptation, we used 360 adaptation trials.

The $30 \%$ size change in shortening and lengthening processes produced different absolute sizes in the two conditions. The average difference from one postsaccadic bar to the next larger one in the shortening condition was $\sim 0.26 \mathrm{deg}$, whereas it was $\sim 0.49 \mathrm{deg}$ in the lengthening condition. Both experiments consisted of the execution first of the task depicted in Figure $2 A$, then of that in Figure 2B, and last in the again the task of Figure $2 A$. We referred to saccadic paradigms as "shortening" and "lengthening" mechanisms to indicate the size-decreasing and sizeincreasing processes defined by Lavergne et al. (2011) and to indicate the direct correlation with the size of the stimulus and not the gap between index and thumb fingers.

Control experiment with verbal report. We further conducted a control experiment to check whether the size estimation effect was specific to manual report or more generally related to size perception. In this experiment, subjects had to indicate the perceived size of the bars verbally as a number in millimeters. Participants sat in front a screen monitor (Eizo FlexScan F930) at $57 \mathrm{~cm}$ that limited a visual field of $40 \times 30 \mathrm{deg}$. The display resolution and the frame rate were $1152 \times$ 864 pixels and $100 \mathrm{~Hz}$, respectively. The stimuli and structure of the experiment were identical to the aforementioned experiments, except for the two estimation phases that consisted of a verbal report of estimated size of presented bars. Each of 11 participants was requested to perform two experimental sessions differentiated by the saccadic adaptation phase and constructed in the same way as Experiments 1 and 2. In one session, subjects executed the shortening adaptation and in the other lenghtening adaptation. These sessions were run on different days with at least $2 \mathrm{~d}$ of separation. During preestimation and postestimation phases, an acoustic signal informed the subject to express a verbal judgment in millimeters about the bar presented on the screen. Each session subjects were presented with a ruler to remind them of the scale. Blue and green colors of stimuli indicated the no saccade and saccade condition, respectively. Number of trials was the same as in Experiment 2.

In all experiments, sequence of bar sizes and conditions were randomly created by MATLAB code. No indication about eye landing position on the bar was provided. All participants received the same instructions.

Data analysis. After data collection, finger position data were interpolated at $1000 \mathrm{~Hz}$ to synchronize with the eye tracker data, then data were run though a fifth-order Butterworth low-pass filter (cutoff frequency, $30 \mathrm{~Hz}$ ). For data processing and analysis, we wrote custom software in MATLAB to compute the distance between index and thumb markers during the manual estimation phases. Grip aperture was calculated considering trial intervals in which the velocities of the index and thumb markers remained $<5 \mathrm{~mm} / \mathrm{s}$. Grip aperture was defined as maximum distance within this interval. We calculated the amplitude of each saccade by determining gaze position directly before the saccade onset (i.e., at the time when the target was presented) and after the saccade reached its ending position. To test successful adaptation, we compared saccadic amplitude between the 20 preadaptation trials and the last 20 adaptation trials (late adaptation). We tested for significance by using two-tailed $t$ tests with independent samples. The amount of shift in saccade amplitude after adaptation was computed as the difference of amplitudes between late adaptation and preadaptation trials averaged across sizes and subjects. We also compared the eye ending position in preadaptation and late adaptation trials as the mean percentage of postsaccadic bar length as follows: Sacc ampl $\times 100 /$ shortened bar length, for shortening adaptation and Sacc ampl $\times 100 /$ lengthened bar length, for lengthening adaptation, respectively. We compared the percentages of landing positions by two-tailed $t$ tests. We used an ANOVA two-way analysis to evaluate the effect of phase (Factor 1) and size (Factor 2) on saccade amplitude and grip aperture and their interaction. The magnitude of change in perceived bar size between preadaptation and postadaptation manual phases was calculated as the difference between postadaptation grip aperture and preadaptation grip aperture. We calculated this deviation averaging across subjects and bar sizes.
The analyses of the verbal report experiment were run on averaged saccade amplitudes and verbal judgments calculated across subjects. To evaluate the amount of adaptation, we extracted the mean deviation from baseline for both variables during shortening and lengthening adaptation, respectively. We compared saccade amplitudes between preadaptation and late adaptation by a two-tailed $t$ test within the shortening and lengthening tasks. We assessed shifts in size perception computing mean deviation from baseline and statistically comparing the postestimation phases during shortening adaptation with that during lengthening adaptation (two-tailed $t$ test).

For all tested participants in manual and verbal experiments for shortening and lengthening adaptation, we calculated correlation coefficient and linear regression between the amplitude of adapted saccades and the size of the postestimation phase. For all statistical analyses, the significance criterion was set to $p<0.05$.

\section{Results}

In Experiments 1 and 2, we performed shortening and lengthening saccadic adaptation toward spatially extended objects and evaluated the change in size perception by hand grip aperture. After adaptation, we measured the adaptation-induced change in saccade amplitude and endpoint as well as grip aperture. Nine of the 14 participants successfully adapted the saccade amplitude in shortening adaptation, and 7 of those 9 participants showed a significant effect on grip aperture. In lengthening adaptation, 9 of the 10 participants displayed significant modification of saccade amplitude and 7 of those showed grip aperture changes. Five of 14 and 1 of 10 did not show any effect on saccade amplitude and grip aperture in shortening and lengthening experiments, respectively.

\section{Saccadic adaptation induced by manipulation of object size}

Before calculating the saccade amplitudes in all trials of the saccade adaptation phase, we checked that the start positions at the beginning of the adaptation phase and those at the end of the adaptation phase did not differ. This ensures that any amplitude change could be ascribed to landing point modifications rather than a shift of the participant's fixation position inside the tracker window in the direction of the future position of the saccade target. There was no significant difference between the mean fixation position in the first and the last 10 trials in all participants. Figure $3 A$ (top) shows saccade amplitudes of one subject over a single session of shortening adaptation of Experiment 1. The first 20 trials were preadaptation delayed saccades where the subject performed saccades to a target bar after an acoustic cue. The target remained at its position and did not change in size during the saccade. The subsequent trials were adaptation trials in which the bar was horizontally shortened during the saccade execution. In the example participant, the saccade amplitude decreased gradually over the adaptation period (Fig. $3 A$, top). Comparison between the average amplitudes before and after adaptation (Fig. $3 A$, bottom) showed a significant shortening of saccade amplitude from $17.03 \pm 0.94 \mathrm{deg}$ to $15.06 \pm 0.65 \mathrm{deg}(t$ test, $p<0.05$ ). Participant B in Figure $3 B$ (top) from the lengthening adaptation group of Experiment 2 showed an increase in amplitude, which resulted in saccade amplitudes that rose from $16.45 \pm 0.94 \mathrm{deg}$ during the preadaptation trials to $19.15 \pm 1.59$ deg in the late adaptation trials (Fig. 3B, bottom). As explained in Materials and Methods, we used a greater number of trials for the lengthening adaptation with respect to the shortening adaptation because saccadic shortening is usually much faster and less variable than saccadic lengthening (Hopp and Fuchs, 2004; Pélisson et al., 2010; Gremmler et al., 2014). 
Average results from all subjects of both groups are presented in Figure 4. Figure $4 A$ shows the amount of adaptation for shortening (in white) and lengthening adaptation (in black) as the difference between the average saccade amplitudes in the baseline trials (preadaptation) and the average saccade amplitudes in the late adaptation trials. Shortening adaptation reached a negative mean value of $-1.17 \pm$ 0.35 . Lengthening adaptation developed very similarly to shortening adaptation but in the opposite direction. In this condition, subjects tended to reach a mean value of $1.36 \pm 0.63$. Figure $4 B$ presents adaptation effects for each bar target. It shows the amplitudes after adaptation as function of the eccentricity of the outer edge of the postsaccadic bar (i.e., that edge of the bar that changed during the saccade where we highlighted the direct correlation of saccadic amplitude changes with the final bar size). The postsaccadic amplitude was size-dependent in both adaptation mechanisms. In the shortening experiment (white dots), the values ranged from $14.92 \mathrm{deg}$ for the smallest bar to 15.82 deg for the biggest one. In lengthening adaptation (black dots), on the other hand, the postsaccadic amplitude grew from 16.17 deg to 18.74 deg according to bar size. A within-group two-way ANOVA (Factor $1=$ phase; Factor $2=$ size) showed main effects of phase and size in shortening adaptation (phase: $F=$ 93.31; $p<0.05$; size: $F=4.15$; $p<0.05)$, but not interaction $(F=0.27, p=0.98)$. Similar results were visible in lengthening adaptation where a significant influence of phase and size was detected (phase: $F=$ 40.61; $p<0.05$; size: $F=2.99$; $p<0.05$ ). The interaction between the two main factors was not statistically significant $(F=$ $0.79, p=0.63$ ).

Next, we investigated where eyes landed on the object before and after adaptation. Figure 5A (left) shows the percentages of bar length where saccades landed averaged over subjects and expressed as percentage of postsaccadic bar length before (left) and after (right) shortening adaptation. In the first case, gaze on average landed at $41.7 \%$ of the postsaccadic bar. After adaptation, gaze landed at $29.7 \%$ of the now even shorter bars indicating a significant shift (two-tailed paired $t$ test, $p<0.05$ ) of landing position toward the inner edge of the target stimulus. The size-specific analysis of eye endpoints run on these data (Fig. 5A, right) showed that, after adaptation, the eye landing position was left-shifted in all bar target sizes. In the lengthening experiment, the landing eye position during preadaptation trials corresponded to $26.7 \%$ of the postsaccadic bar. After adaptation,

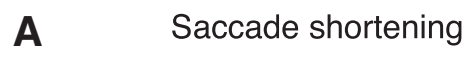

\section{B Saccade lengthening}
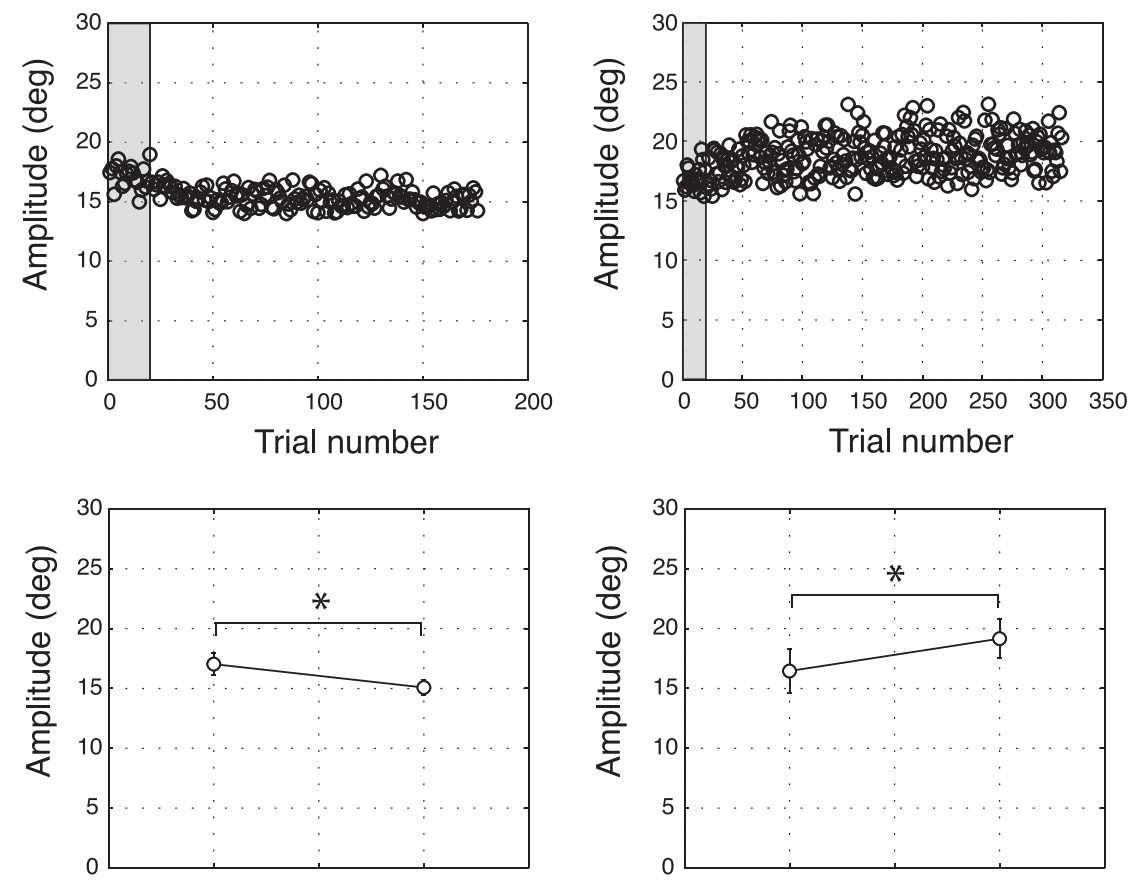

Pre-adaptation Late-adaptation



Pre-adaptation Late-adaptation

Figure 3. Example sessions. A, Shortening saccades. Top, Saccade amplitudes of an example participant from the shortening group. Saccades executed during preadaptation trials are presented within the gray shadow. Bottom, Average amplitude of preadaptation and late adaptation trials of the example participant. $\boldsymbol{B}$, Top, Saccade amplitude of a participant of the lengthening group. Saccades executed during preadaptation trials are presented within the gray shadow. Bottom, Average amplitude of preadaptation and late adaptation trials of the example participant. Error bars indicate SE. ${ }^{*} p<0.05$, significant level.

\section{A Average saccade amplitude deviation}

B

Saccade amplitude


Shortening adaptation

Lengthening adaptation

Figure 4. A, Mean deviation of saccade amplitude from baseline for shortening adaptation (white column) and lengthening adaptation (black column) averaged across subjects and sizes. $\boldsymbol{B}$, Mean saccade amplitude for shortening (white dots) and lengthening adaptation (black dots) averaged across subjects and plotted as function of eccentricity of postsaccadic outer edge of bar. Error bars indicate SE. ${ }^{*} p<0.05$, significant level.

the landing eye position was quite precise in matching the central location of the stimulus (Fig. $5 B$, left). Indeed, the average endpoint corresponded to $44.6 \%$ of stimulus length, and this pattern was similar for all bar sizes (Fig. 5B, right). In shortening session, the absolute deviation of eye landing position between preadap- 


\section{A}

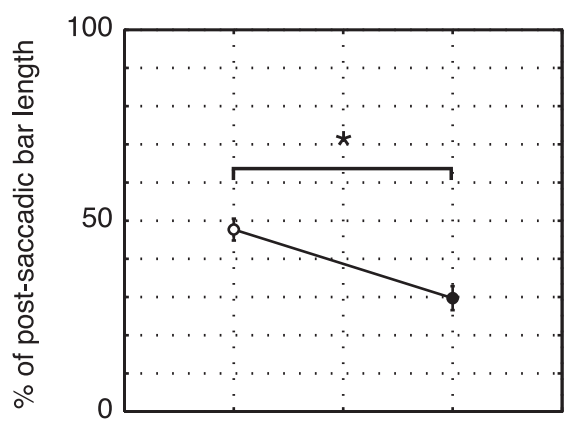

Pre-adaptation Late-adaptation

Pre-adaptation
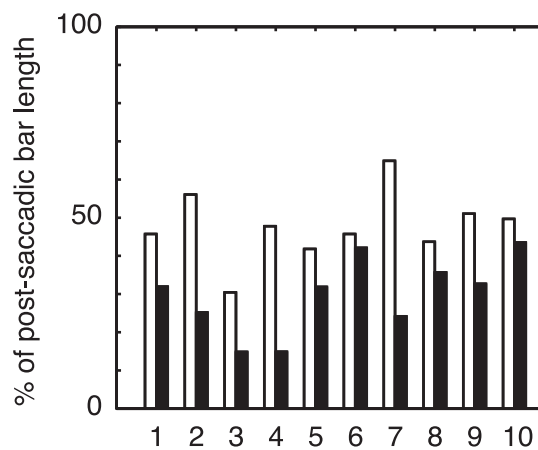

Bar size

Late-adaptation
B



Lengthening adaptation

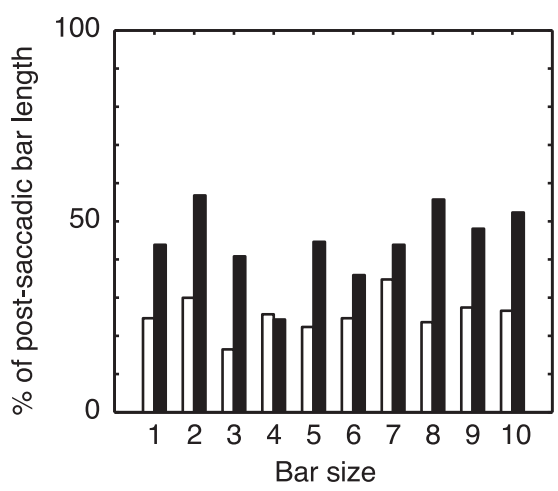

Figure 5. Landing points of the saccades calculated as percentage of postsaccadic bar length. $\boldsymbol{A}$, Shortening adaptation. Left, Mean landing position of preadaptation trials (white) and adaptation trials (black) averaged across subjects and sizes. Right, Mean landing position of preadaptation trials (white) and late adaptation trials (black) averaged for each bar. $\boldsymbol{B}$, Lengthening adaptation. Left, Mean landing position of preadaptation trials (white) and late adaptation trials (black) averaged across subjects and sizes. Right, Mean landing position of preadaptation trials (white) and late adaptation trials (black) averaged for each bar. Error bars indicate $S E$. ${ }^{*} p<0.05$, significant level.

tation and adaptation eye movements was $\sim 12 \%$, whereas in lengthening adaptation this deviation corresponded to $18 \%$.

Although the average endpoints of preadaptation trials are different in the two groups, being the lengthening group more hypometric, the preadaptation amplitudes for each bar size were not different between shortening and lengthening group (twotailed paired $t$ test, $p>0.05)$. Furthermore, individual data showed that the most hypometric participant from the shortening group presented a significant saccade amplitude reduction (from 15.45 to $14.36 \mathrm{deg}, p<0.05$ ) as well as the most hypermetric participant from lengthening group significantly extended the saccade amplitude after adaptation (from 17.26 to $18.69 \mathrm{deg}, p<$ $0.05)$. We can conclude with confidence that the observed shifts were due to adaptation and not to changes in the baseline among the two groups of participants. The combination of amplitude and endpoint analysis clearly shows that saccadic adaptation can be induced by modifying target size. Indeed, the progressive adjustment of saccade amplitudes and endpoints led to the reestablishing of the accuracy in landing inside the spatially extended target with the primary saccade.

\section{Object size misperception}

The second goal of the present study was to test for a change in visual size perception of the peripherally presented stimuli. We asked participants of the two groups of Experiments 1 and 2, before and after the adaptation phase, to indicate with the aperture of the thumb and index fingers (grip aperture) the perceived horizontal size of the presented bar. We did so in two conditions: one in which the subject had to keep fixation (called no saccade condition) and the other (called saccade condition) in which the subject made a saccade to the peripheral bar but the bar was extinguished during the saccade. Thus, the subject in both conditions only saw the presaccadic bars, but the conditions differed in terms of whether the subject planned and performed a saccade or not. A within-group $t$ test analysis showed no significant differences (shortening group, $p=0.48$; lengthening group, $p=0.54$ ) in the grip apertures between the two conditions. Thus we analyzed the results collectively.

Figure $6 A$ shows the average amount of misperception of object size after shortening adaptation (white column) and lengthening adaptation (black column) in all subjects tested. The modification of visual size perception reached a mean value of $-3.89 \pm 3.35 \mathrm{~mm}$ after shortening adaptation and a mean value of $5.55 \pm 3.83$ $\mathrm{mm}$ after lengthening adaptation.

To evaluate the effect of phase and size on grip aperture, we performed a two-way ANOVA (Factor 1, Phase: 2 levels; Factor 2, size: 10 levels). The adaptation phase had a clear effect on visual perception of object size. In the group that executed shortening adaptation, significant main effects of phase and size were observed (phase: $F=16.29$; $p<0.05$; size: $F=16.76 ; p<0.05$ ). A slight decrease of finger distance after the adaptation phase in the longer bars compared with the baseline was observed and reached statistical significance only for the highest bar size (Fig. 6B, white dots; post hoc test with Tukey's correction). Similarly and in the opposite direction, the subjects of the group tested in lengthening adaptation presented significant main effects of phase and size factors (phase: $F=19.25 ; p<0.05$; size: $F=19.02 ; p<0.05$ ) and an increase of grip aperture after adaptation in all bar sizes that showed statistical difference compared with the baseline for some of the longer bars (Fig. 6B, black dots; post hoc test with Tukey's correction). Overall, these results demonstrate a change of size perception for peripheral objects that followed the direction of saccadic adaptation. In the shortening group, the modification of visual perception was observable only for the longer bars. The baseline shown in Figure $6 B$ (dashed line) was the result of an average across the pregrip apertures of the two groups (pooled together because they did not display significant differences; twopaired $t$ test for each bar size, $p>0.05$ ). The statistical effect of size indicates a good ability of participants to discriminate among 
different object sizes as it is observable by the linear pattern of grip apertures distribution. There was no significant interaction between phase and size factors in both groups.

To compare object size perception changes after adaptation, we calculated the mean deviation of grip apertures from preestimation baseline trials. The size perception change was calculated similarly to saccade amplitude deviation, as the difference between mean grip aperture in the preestimation baseline trials and mean grip aperture in the block of postestimation trials. The development of perceptual modification in eye and hand domains was partially similar. Indeed, the dependence on object size of saccade amplitude and grip aperture was evident in both shortening and lengthening adaptation because the two parameters showed the same linear distribution according to the size (Figs. $4 B, 6 B$ ).
A Average grip aperture deviation

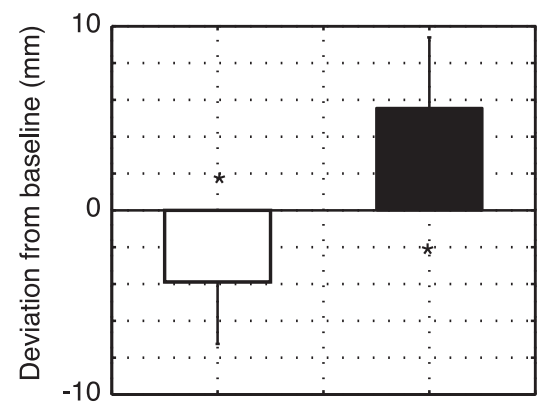

Shortening adaptation
Lengthening adaptation
B

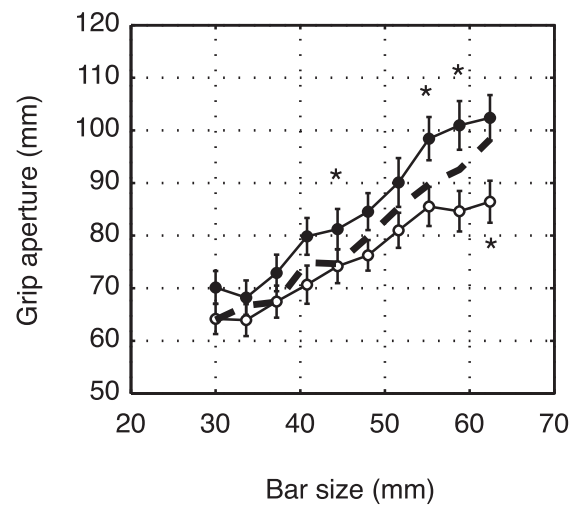

Shortening adaptation

Lengthening adaptation

Baseline

Figure 6. A, Mean deviation of grip aperture from baseline for shortening adaptation (white column) and lengthening adaptation (black column) averaged across subjects and sizes. $\boldsymbol{B}$, Distribution of mean postgrip apertures for shortening and lengthening adaptation (white and black dots, respectively). Dotted line indicates the baseline (pregrip apertures) averaged across two groups. Error bars indicate SE. ${ }^{*} p<0.05$, significant deviations from baseline (dashed line).

\section{Control experiment with verbal reports of perceived size}

Similar changes in object size perception were observed in the participants executing the verbal report before and after saccadic adaptation. No significant differences were found in the preestimation phases of no saccade and saccade condition across shortening and lengthening sessions, so we averaged all values together. The average amount of shift after shortening adaptation was $-2.26 \pm 1.31 \mathrm{~mm}$ and after lengthening adaptation was $1.64 \pm 1.02 \mathrm{~mm}$. We found statistical differences between postshortening and postlengthening estimation phase (two-tailed $t$ test, $p<0.05$ ) indicating a change in visual perception in the direction of saccadic adaptation also when participants used the verbal modality to report size features of targets. Averaged adaptation of saccade amplitudes in this experiment was $-0.21 \pm 0.19 \mathrm{deg}(t$ test, $p<0.05)$ in the shortening session and $0.65 \pm 0.5 \mathrm{deg}$ ( $t$ test, $p<0.05)$ in the lengthening session.

To further confirm whether saccadic adaptation induced size misperception, we performed a linear regression and a correlation analysis between the individual amplitudes of adapted saccades and the size estimates after saccadic adaptation of manual and verbal experiments pooling together the shortening and lengthening adaptations. Figure 7 shows a linear relationship $\left(r^{2}=0.2\right)$ between the two variables confirming that changes in saccade amplitude were transferred to size perception ability. The two variables showed a significant correlation coefficient $(r=$ $0.44, p<0.05$ ).

\section{Discussion}

Our study is the first to systematically adapt the amplitude of saccades to spatially extended targets and test the change of visual perception of the horizontal dimension of the same targets.

Saccadic adaptation may be induced by changing object size Decreasing or increasing object size during saccade execution led to a gradual adaptive modification in the amplitude of saccades with a concomitant shift of saccade landing position in the direc-

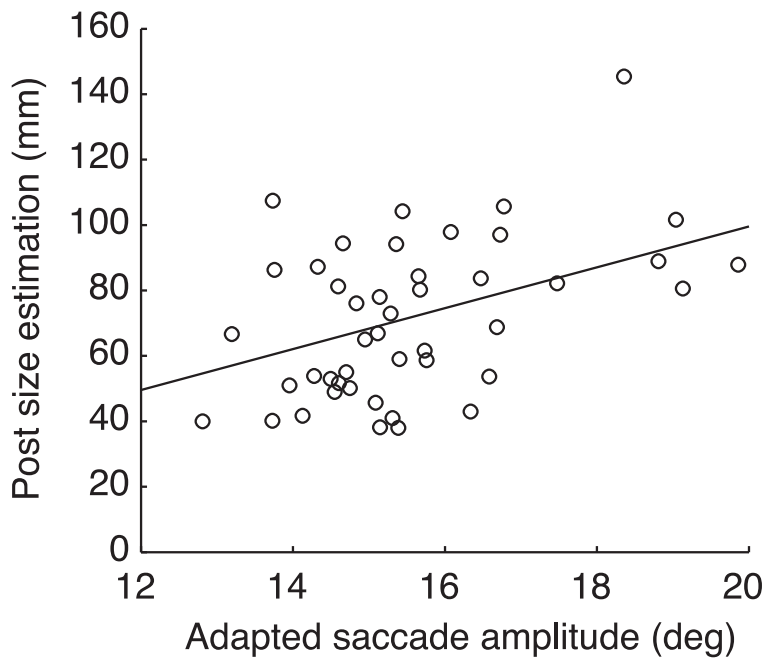

Figure 7. Postadaptation size estimates as function of postadaptation saccade amplitudes and linear regression analysis. Each point corresponds to each participant tested in manual and verbal experiments and in shortening and lengthening adaptation. Line represents linear regression. The equation of line is $y=6.2 x-25\left(r^{2}=0.2\right)$. Correlation coefficient is $0.44(p<0.05)$.

tion of the size change. Lengthening adaptation was stronger in our subjects than shortening adaptation. This may have occurred in Experiments 1 and 2 because we used a larger number of trials in the lengthening experiment because, classically, shortening adaptation develops quicker than to lengthening adaptation in reactive saccades (Deubel et al., 1986; Straube et al., 1997, Panouillères et al., 2009). However, the stronger lengthening adaptation was also apparent in the verbal report experiment, which used the same number of trials for both lengthening and shortening conditions. Thus, it rather seems that adaptation to the manipulation of the size of extended saccade target has somewhat different properties than adaptation to the shift of a small target.

Saccades directed to spatially extended targets, such as random-dot clusters or simple shapes, have been shown to land at 
consistent location near the center of the target area regardless of target size (He and Kowler, 1991; Kowler and Blaser, 1995; McGowan et al., 1998; Melcher and Kowler, 1999). The saccade endpoint distribution in our experiment confirms that the saccadic system has access to a reference position within spatially extended targets (Kowler and Blaser, 1995; McGowan et al., 1998) that is maintained during adaptation process and is highly correlated with saccade amplitude. Indeed, the percentage of shift in both adaptation directions tested $(\sim 12 \%$ and $18 \%$ in shortening and lengthening adaptation of Experiments 1 and 2, respectively) suggests that the oculomotor system updates the landing position representation of the stimulus.

In our study, we manipulated an intrinsic property of the target, namely, its size. Earlier attempts to adaptively lengthen the amplitude of saccades aiming for a target of a given shape while simultaneously shortening the amplitude of saccades aiming for another shape did not produce shape-specific adaptation (Bahcall and Kowler, 2000). The intrinsic target property shape is not taken into account for plastic modifications of saccadic system. Object size is also an intrinsic visual property; but unlike shape, size can be critical for the coding of the saccades. In our experiment, subjects were simply instructed to look at the target without receiving any other indication about the saccade ending position. Furthermore, the only parameter that changed was the target length maintaining constant the shape; thus, the alteration of saccadic amplitude observed could be the result of computations extracted by stimulus size information together with its position (Findlay, 1982; Lavergne et al., 2010). In our study, the dependence of adaptation on target size was clearly visible in shortening and lengthening adaptation mechanisms. Indeed, the saccade amplitudes showed a distribution as function of target size (Fig. $4 B$, white and black dots). One possible explanation is the visual distance between the saccade endpoint and the outer edge of the object. In this view, the saccadic system could predict how far the eyes should arrive from the object-end and compare it with the actual distance in the adaptation trials. Another explanation may be that size information about the object is encoded before the saccade and then compared with the postsaccadic object. Adaptation could be the result of a mechanism that compares presaccadic and postsaccadic sizes. In an exploring saccade study, Vergilino and Beauvillain (2000) presented a letter string that was shortened or lengthened at different times after the first saccade toward the string, and they showed that the saccadic system was able to recalibrate the motor vector of the saccade if the change was intervening very early before the execution of the exploring saccade. This suggests that information about object size may be updated after or during the first saccade execution. Lavergne et al. (2011) showed that classical targeting saccade adaptation did not transfer to exploring saccades, but the reciprocal transfer (from exploring to targeting saccade) occurred when targeting saccades aimed for a spatially extended stimulus. They proposed that targeting saccades to a spatially extended stimulus were coded not only as a function of stimulus position but also as a function of stimulus size, as they are directed to the center of gravity (Findlay, 1982; Vergilino and Beauvillain, 2001). In our study, we adapted primary saccades and not exploring saccades, and we observed that gaze landed always close to a middle reference position within the stimulus. However, the calculation of this representative position inside the stimulus takes into account the object size.

\section{Size perception is affected by saccadic adaptation}

Our data further show that the perceptual indication of object size by grip aperture changed after saccadic adaptation, regardless of whether the subjects performed a saccade to the object or not. The grip aperture change implies a link between saccade motor parameters and visual perception of object size. This deformation of the perceptual representation of object size is novel in the literature and partially follows the same dynamics of target localization perception. Many earlier studies focused on the transfer of saccadic modification to other domains. Both Bekkering et al. (1995) and Kröller et al. (1996) found an alteration of the goaldirected hand movement for regions close to the saccadic target. More recently, different studies (Bruno and Morrone, 2007; Cotti et al., 2007) demonstrated a substantial transfer of adaptation in pointing localization. Also, perceptual reports of target position for both gain-decreasing and gain-increasing conditions suggested influences on perceptual and action representation (Bahcall and Kowler, 1999; Awater et al., 2005; Collins et al., 2007; Gremmler et al., 2014). Zimmerman and Lappe (2010) concluded that oculomotor errors induce not only adaptations of the motor commands but also affect visual perception of location. Garaas and Pomplun (2011) found that simultaneous adaptation of saccades in different directions can induce a change in perceived object shape. In our experiments, we modified the visual size perception of objects by a saccadic adaptation paradigm based on changes in stimulus size. The distortion of perceptual stimulus size observed here in manual and verbal modality is evidence that the saccadic system bases the eye movement on object extension and transfers this knowledge to systems encoding this signal to recognize and/or grasp the object. The correlation analysis (Fig. 7) confirms that misperception of object size is related to saccadic adaptation. During the adaptation phase, participants were exposed to two views of the target bar: a peripheral view of a particular size and a parafoveal view of a different size. Our results show that the size perception of the peripheral view is modified by the size of the later parafoveal view. Given the vastly different resolution between peripheral and foveal vision, it may be beneficial for the visual system to use the parafoveal view of an object that one has just reached with a saccade to calibrate size perception in the visual periphery. If object correspondence is established across the saccade, the current size of the object may be associated with the previous peripheral view to tune peripheral size perception for future targets. Similar learned associations between peripheral and foveal representations of spatial frequency have recently been demonstrated by Herwig and Schneider (2014). Such associations can be used for predictions of peripheral or foveal input to deal with the nonhomogeneity of the visual system. This means that, in our case, peripheral information that is associated with a foveal template due to previous trans-saccadic experience is used to predict the related sensory peripheral appearance. Thus, it is possible that participants used previously associated peripheral or foveal visual input to predict how object features such as size will look like in the periphery or in the fovea.

The importance of parafoveal vision to recognize and perform movements to objects was established by several studies (i.e., on face processing and hand/eye coordination; Johansson et al., 2001). In peripheral vision, face recognition is limited compared with central vision (Levy et al., 2001; Hasson et al., 2002; Loomis et al., 2008). This is because in peripheral vision spatial acuity is reduced (Anstis, 1974) and there is increased crowding between features (Pelli and Tillman, 2008). Furthermore, it was demonstrated that fixation of visual targets increases reaching- 
movement accuracy (Prablanc et al., 1979; Bock, 1986; Neggers and Bekkering, 1999; van Donkelaar and Staub, 2000). In the present study, we found a substantial transfer of size visual misperception to grip aperture. This pattern is compatible with the view that object features such as size are better processed when the eyes capture the target with the fovea. Typically, the grip aperture is a parameter highly correlated with target size during grasping movements. In such view, we can argue that the distortion of grip aperture following the saccadic adaptation is strongly based on parafoveal vision. Indeed, grasping and manipulation of objects require a representation of the central part of visual field that is critical to collect visual information for the interaction between hand and object.

\section{References}

Anstis SM (1974) Letter: a chart demonstrating variations in acuity with retinal position. Vision Res 14:589-592. Medline

Awater H, Burr D, Lappe M, Morrone MC, Goldberg ME (2005) Effect of saccadic adaptation on localization of visual targets. J Neurophysiol 93: 3605-3614. CrossRef Medline

Bahcall DO, Kowler E (1999) Illusory shifts in visual direction accompany adaptation of saccadic eye movements. Nature 400:864-866. CrossRef Medline

Bahcall DO, Kowler E (2000) The control of saccadic adaptation: implications for the scanning of natural visual scenes. Vision Res 40:2779-2796. CrossRef Medline

Bekkering H, Adam JJ, van den Aarssen A, Kingma H, Whiting HT (1995) Interference between saccadic eye and goal-directed hand movements. Exp Brain Res 106:475-484. CrossRef Medline

Bock O (1986) Contribution of retinal versus extraretinal signals towards visual localization in goal-directed movements. Exp Brain Res 64:476482. CrossRef Medline

Brainard DH (1997) The Psychophysics Toolbox. Spat Vis 10:433-436. CrossRef Medline

Bruno A, Morrone MC (2007) Influence of saccadic adaptation on spatial localization: comparison of verbal and pointing reports. J Vis 7:16.11-13. CrossRef Medline

Collins T, Doré-Mazars K, Lappe M (2007) Motor space structures perceptual space: evidence from human saccadic adaptation. Brain Res 1172: 32-39. CrossRef Medline

Cotti J, Guillaume A, Alahyane N, Pélisson D, Vercher JL (2007) Adaptation of voluntary saccades, but not of reactive saccades, transfers to hand pointing movements. J Neurophysiol 98:602-612. CrossRef Medline

Deubel H, Wolf W, Hauske G (1986) Adaptive gain control of saccadic eye movements. Hum Neurobiol 5:245-253. Medline

Ethier V, Zee DS, Shadmehr R (2008) Spontaneous recovery of motor memory during saccade adaptation. J Neurophysiol 99:2577-2583. CrossRef Medline

Findlay JM (1982) Global visual processing for saccadic eye movements. Vision Res 22:1033-1045. CrossRef Medline

Garaas TW, Pomplun M (2011) Distorted object perception following whole-field adaptation of saccadic eye movements. J Vis 11:2. CrossRef Medline

Golla H, Tziridis K, Haarmeier T, Catz N, Barash S, Thier P (2008) Reduced saccadic resilience and impaired saccadic adaptation due to cerebellar disease. Eur J Neurosci 27:132-144. CrossRef Medline

Gremmler S, Bosco A, Fattori P, Lappe M (2014) Saccadic adaptation shapes visual space in macaques. J Neurophysiol 111:1846-1851. CrossRef Medline

Hasson U, Levy I, Behrmann M, Hendler T, Malach R (2002) Eccentricity bias as an organizing principle for human high-order object areas. Neuron 34:479-490. CrossRef Medline
He PY, Kowler E (1991) Saccadic localization of eccentric forms. J Opt Soc Am A 8:440-449. CrossRef Medline

Herwig A, Schneider WX (2014) Predicting object features across saccades: evidence from object recognition and visual search. J Exp Psychol Gen 143:1903-1922. CrossRef Medline

Hopp JJ, Fuchs AF (2004) The characteristics and neuronal substrate of saccadic eye movement plasticity. Prog Neurobiol 72:27-53. CrossRef Medline

Johansson RS, Westling G, Bäckström A, Flanagan JR (2001) Eye-hand coordination in object manipulation. J Neurosci 21:6917-6932. Medline

Kowler E, Blaser E (1995) The accuracy and precision of saccades to small and large targets. Vision Res 35:1741-1754. CrossRef Medline

Kröller J, Pélisson D, Prablanc C (1996) On the short-term adaptation of eye saccades and its transfer to head movements. Exp Brain Res 111:477-482. Medline

Lavergne L, Vergilino-Perez D, Collins T, Doré-Mazars K (2010) Adaptation of within-object saccades can be induced by changing stimulus size. Exp Brain Res 203:773-780. CrossRef Medline

Lavergne L, Vergilino-Perez D, Lemoine C, Collins T, Doré-Mazars K (2011) Exploring and targeting saccades dissociated by saccadic adaptation. Brain Res 1415:47-55. CrossRef Medline

Levy I, Hasson U, Avidan G, Hendler T, Malach R (2001) Center-periphery organization of human object areas. Nat Neurosci 4:533-539. Medline

Loomis JM, Kelly JW, Pusch M, Bailenson JN, Beall AC (2008) Psychophysics of perceiving eye-gaze and head direction with peripheral vision: implications for the dynamics of eye-gaze behavior. Perception 37:14431457. CrossRef Medline

McConkie GW, Kerr PW, Reddix MD, Zola D, Jacobs AM (1989) Eye movement control during reading: II. Frequency of refixating a word. Percept Psychophys 46:245-253. CrossRef Medline

McGowan JW, Kowler E, Sharma A, Chubb C (1998) Saccadic localization of random dot targets. Vision Res 38:895-909. CrossRef Medline

Melcher D, Kowler E (1999) Shapes, surfaces and saccades. Vision Res 39: 2929-2946. CrossRef Medline

Neggers SF, Bekkering H (1999) Integration of visual and somatosensory target information in goal-directed eye and arm movements. Exp Brain Res 125:97-107. CrossRef Medline

Panouillères M, Weiss T, Urquizar C, Salemme R, Munoz DP, Pélisson D (2009) Behavioral evidence of separate adaptation mechanisms controlling saccade amplitude lengthening and shortening. J Neurophysiol 101: 1550-1559. CrossRef Medline

Pélisson D, Alahyane N, Panouillères M, Tilikete C (2010) Sensorimotor adaptation of saccadic eye movements. Neurosci Biobehav Rev 34:11031120. CrossRef Medline

Pelli DG, Tillman KA (2008) The uncrowded window of object recognition. Nat Neurosci 11:1129-1135. CrossRef Medline

Prablanc C, Echallier JF, Komilis E, Jeannerod M (1979) Optimal response of eye and hand motor systems in pointing at a visual target: I. Spatiotemporal characteristics of eye and hand movements and their relationships when varying the amount of visual information. Biol Cybern 35: 113-124. CrossRef Medline

Straube A, Robinson FR, Fuchs AF (1997) Decrease in saccadic performance after many visually guided saccadic eye movements in monkeys. Invest Ophthalmol Vis Sci 38:2810-2816. Medline

van Donkelaar P, Staub J (2000) Eye-hand coordination to visual versus remembered targets. Exp Brain Res 133:414-418. CrossRef Medline

Vergilino D, Beauvillain C (2000) The planning of refixation saccades in reading. Vision Res 40:3527-3538. CrossRef Medline

Vergilino D, Beauvillain C (2001) Reference frames in reading: evidence from visually and memory-guided saccades. Vision Res 41:3547-3557. CrossRef Medline

Zimmermann E, Lappe M (2010) Motor signals in visual localization. J Vis 10:2. CrossRef Medline 